\title{
ANÁLISE DE COVARIÂNCIA PARA MELHORIA DA CAPACIDADE DE DISCRIMINAÇÃO EM ENSAIOS DE CULTIVARES DE MILHO'
}

\author{
LINDOLFO STORCK², SIDINEI JOSÉ LOPES ${ }^{3}$, DANIELA GIFFONI MARQUES ${ }^{4}$, \\ CESAR AUGUSTO TISOTT ${ }^{5}$ e CESARAUGUSTO DAROS ${ }^{6}$
}

\begin{abstract}
RESUMO - $\mathrm{O}$ uso de técnicas experimentais visando à precisão em detectar diferenças no rendimento de grãos é muito importante. O objetivo deste trabalho foi verificar a viabilidade da análise da covariância, como forma de reduzir o erro experimental. Foram utilizados os dados de rendimento de grãos de milho (Zea mays L.) e da população final de plantas das unidades experimentais de 313 ensaios de competição de cultivares, conduzidos no Estado do Rio Grande do Sul nos anos de 1993/96. Para fins de comparação entre a precisão da análise com e sem o uso da covariância, criou-se uma estatística denominada HD, que é igual à amplitude entre as médias estimadas das cultivares, dividida pelo valor da diferença mínima significativa (DMS) do teste de Tukey. Quanto maior for o valor de HD, melhor será a qualidade do ensaio, por permitir melhor discriminação entre as cultivares. $O$ uso da análise da covariância elevou a estatística HD de 4,7 para 19,5, e melhorou a discriminação entre as médias ajustadas pela covariável população final de plantas.
\end{abstract}

Termos para indexação: Zea mays, rendimento de grãos, população de plantas, análises estatísticas.

\section{COVARIANCE ANALYSIS TO IMPROVE DISCRIMINATION CAPACITY IN CULTIVARS' CORN TRIALS}

\begin{abstract}
The use of experimental techniques in order to increase trial precision is important to detect small yield gain in crops. The aim of this research was to evaluate the potential use of covariance analyses to increase trial precision. Data of grain yield and final plant population from 313 corn (Zea mays L.) cultivar trials were studied. The experiments were carried out under different environmental conditions of the state of Rio Grande do Sul, Brazil, from 1993 to 1996. An HD statistic to compare the precision for use and not use of the covariance analyses was developed. The HD is the amplitude among cultivar mean estimations divided by the LSD value of Tukey test. The highest HD value, the best trial precision is obtained, which permits to detect small differences between cultivars. It was verified that covariance analyses increased HD from 4.7 to 19.5 improving cultivar discrimination yields adjusted by the covariable final plant population.
\end{abstract}

Index terms: Zea mays, grain yield, plant population, statistical analysis.

\section{INTRODUÇÃO}

Das técnicas experimentais usadas na pesquisa depende, em grande parte, a qualidade das informa-

${ }^{1}$ Aceito para publicação em 2 de dezembro de 1999.

${ }^{2}$ Eng. Agrôn., Dr., Prof. Titular, Dep. de Fitotecnia, Universidade Federal de Santa Maria (UFSM), CEP 97105-900 Santa Maria, RS. Bolsista do CNPq.

E-mail: storck@ccr.ufsm.br

${ }^{3}$ Eng. Agôn., M.Sc., Prof. Assistente, Dep. de Fitotecnia, UFSM. E-mail: sjlopes@ccr.ufsm.br

${ }^{4}$ Eng. Agôn., M.Sc. E-mail: daniela@fcav.unesp.br

${ }^{5}$ Eng. Agôn., UFSM. Bolsista do PIBIC/UFSM/CNPq

${ }^{6}$ Aluno do Curso de Agronomia, UFSM. Bolsista da FAPERGS. ções obtidas. Assim, o planejamento, a execução, a análise e a interpretação adequada dos resultados dos experimentos são etapas fundamentais para o sucesso da pesquisa (Storck \& Lopes, 1997). Na etapa da análise é possível identificar problemas ocorridos no planejamento, na execução e na própria análise. Para isto, basta realizar os principais testes sobre a adequação às pressuposições do modelo matemático adotado. A qualidade de um experimento é avaliada pela magnitude do erro experimental, isto é, pela variação entre unidades experimentais submetidas ao mesmo tratamento. Um erro experimental elevado implica não rejeitar a hipótese nula quando efetivamente falsa. 
No planejamento de experimentos são controlados: o tamanho das unidades experimentais, o número de repetições, as restrições na casualização, o número de tratamentos e o manejo a ser empregado. Existem métodos próprios para determinar esses parâmetros (Storck, 1979; Lopes, 1993; Oliveira, 1994, Lopes \& Storck, 1995). Na execução dos experimentos, determinadas técnicas de manejo são fundamentais para a redução do erro experimental, destacando-se: o uso de desbaste, controle de plantas daninhas, doenças e pragas realizados preventivamente, uso de bordadura, material experimental uniforme, tratos culturais homogêneos e concordandes com o delineamento.

A verificação da adequação às pressuposições do modelo é necessária para que os testes de hipóteses sejam exatos e as conclusões confiáveis, e portanto, para análises de qualidade. Muitas vezes, o não-atendimento de alguma pressuposição pode estar associado a práticas culturais, a características dos tratamentos, a uma variável observada, e à forma de avaliação. A identificação de práticas culturais que interferem na análise é importante para uma recomendação de melhoria na qualidade da pesquisa. A baixa precisão experimental dos Ensaios Estaduais de Cultivares de Milho no Rio Grande do Sul já foi ressaltada em trabalhos anteriores (Lopes, 1993; Lopes \& Storck, 1995; Lúcio, 1997), e identificada pelas elevadas diferenças mínimas significativas, em porcentagem da média, entre as cultivares, que, não raramente, ultrapassam $50 \%$ do rendimento de grãos, superando a média de produtividade obtida pelos produtores no Estado. Na prática, as pressuposições sobre os erros do modelo e a aditividade do modelo, necessárias para um adequado desenvolvimento da análise da variância e dos testes de hipóteses, nem sempre são verdadeiras. Como o teste $\mathrm{F}$ da análise da variância não é sensível a alguns desvios das pressuposições, o procedimento empregado, normalmente, é ignorar a verificação das pressuposições. Entretanto, se o desvio for acentuado, os resultados da análise podem ser bastante alterados, comprometendo assim as conclusões (Box \& Cox, 1964; Siqueira, 1983).

Quando se necessita analisar dados que não estão de acordo com as pressuposições do modelo estatístico a ser utilizado, procura-se, normalmente, uma das seguintes alternativas: modificar o método de análise, buscando aquele que melhor se ajusta aos dados (análise não-paramétrica); modificar a variável resposta, adequando-a, através de transformações não-lineares, às pressuposições do modelo. Pimentel-Gomes (1990) ressalta que, mesmo com um grande número de testes não-paramétricos, estes são, geralmente, de aplicação restrita e de resultados pouco satisfatórios, apesar de não exigirem distribuição normal dos erros, nem variâncias homogêneas.

A heterocedasticidade dos erros pode ser conseqüência de dois fatores principais: a natureza dos tratamentos e o planejamento ou manejo do experimento. Assim como a natureza dos tratamentos pode fornecer respostas mais variáveis do que outras, causando heterogeneidade da estimativa da variância, os manejos aplicados aos experimentos podem trazer maior ou menor variabilidade (Lopes \& Storck, 1995), acarretando algumas vezes conclusões incorretas. Siqueira (1983) atribui a falta de cuidados na condução do experimento e a escolha incorreta da unidade experimental como prováveis causas da heterocedasticidade. A heterocedasticidade entre as unidades experimentais pode ser classificada em regular e irregular (Cochran, 1947; Steel \& Torrie, 1960). A regular, geralmente ocorre pela falta de normalidade dos erros e pelo relacionamento entre as médias e as variâncias dos vários tratamentos. Nesse caso, os dados podem ser transformados, para se aproximarem da distribuição normal. A heterocedasticidade caracterizada como irregular surge de tratamentos com variabilidade significativamente maior do que a de outros, sem que necessariamente haja relação entre a média e a variância.

A avaliação da magnitude do erro experimental pode ser feita pelo uso de estatísticas, como a diferença mínima significativa (DMS), a qual é confrontada com limites preestabelecidos, com base em grupos de experimentos semelhantes (Storck \& Lopes, 1997). A qualidade de um experimento em particular é avaliada pelos valores do coeficiente de variação $(\mathrm{CV})$ e da DMS obtida pelo teste de Tukey expresso em porcentagem da média do experimento, quando comparados com os limites das classes previamente estabelecidos (Estefanel et al., 1987; Lúcio, 1997). Quanto à cultura do milho, Lúcio (1997) observou influên- 
cia do desbaste e da adubação em cobertura sobre os valores do CV e da DMS.

O objetivo deste trabalho foi analisar a viabilidade da análise de covariância entre o rendimento de grãos e a população final de plantas para melhorar a capacidade de discriminação entre cultivares de milho.

\section{MATERIAL E MÉTODOS}

Foram utilizados os dados originais de rendimento de grãos de milho (Y) e da população final de plantas (X), por unidade experimental, de 313 ensaios de competição de cultivares conduzidos em diferentes locais do Estado do Rio Grande do Sul, sob a coordenação da FEPAGRO/ RS, durante os anos de 1993 a 1996. Os ensaios são classificados quanto ao ciclo das cultivares (superprecoce, precoce e normal) e quanto à categoria (preliminar, estadual e recomendação). Na totalidade dos ensaios, o delineamento experimental foi o de blocos ao acaso, com número de repetições variando entre três e quatro. Alguns ensaios com duas repetições não foram analisados. O número de cultivares foi de 9 a 42, variando de acordo com o ciclo e a categoria.

Em cada ensaio, as variáveis $\mathrm{X}$ e $\mathrm{Y}$ foram submetidas a uma análise da variância, para verificar a significância do efeito de bloco e de cultivar, e calcular a diferença mínima significativa (DMS) pelo teste de Tukey ( $5 \%$ ) em porcentagem da média do ensaio. DMS $=100 *(\Delta / \mathrm{m})$ para $\Delta=\mathrm{q} \sqrt{\mathrm{QMe} / \mathrm{J}} ; \mathrm{q}$ é o valor da Tabela do teste de Tukey; QMe é o quadrado médio do erro; J é o número de repetições, e m é a média geral do ensaio. Foi calculada a estatística HD da variável Y, que é igual à amplitude entre as médias estimadas das cultivares, dividida pelo valor de $\Delta$ do teste de Tukey. Acredita-se que, quanto maior for o valor de HD, melhor será a qualidade do ensaio, por permitir melhor discriminação entre as cultivares, facilitando a escolha das melhores e a eliminação das piores cultivares. Foram descartados os ensaios em que, para a variável Y, não foi verificada a distribuição normal do erro - pelo teste de Lilliefors (Campos, 1983) - ou a homogeneidade das variâncias dos erros entre as cultivares pelo teste de Bartlett (Steel \& Torrie, 1960).

No estudo da viabilidade do uso da análise da covariância, tomando a variável X como covariável, foi feita análise da covariância, para verificar a significância de $\mathrm{X}$ sobre $\mathrm{Y}$ e o efeito de blocos e de cultivares ajustadas. Esses ensaios também foram analisados sem a covariância, para estimar o efeito de blocos, cultivares, DMS e HD. Para o cálculo da eficiência relativa (ER) do uso da covariância, da DMS ajustada e da estatística HD ajustada, a estimativa da variância das estimativas das médias ajustadas foi ponderada conforme Steel \& Torrie (1960). Foi aplicado o teste de F para testar a hipótese de paralelismo (Seber, 1976) do efeito da covariável entre as cultivares.

\section{RESULTADOS E DISCUSSÃO}

De um total de 313 ensaios, a pressuposição de distribuição normal do erro não foi observada em 14 ensaios $(4,5 \%)$ e a pressuposição da homogeneidade das variâncias dos erros entre cultivares não foi observada em cinco ensaios (1,6\%), apenas dois ensaios não tiveram as duas pressuposições atendidas. No total, foram descartados $5,4 \%$ dos ensaios, por falta de atendimento de pressuposições. Estes ensaios não foram usados para aplicação da covariância visando estimar a eficiência da mesma por se tratar de uma quantidade insegura para se obter uma conclusão.

Quanto à variável população final de plantas (X), observa-se que, entre outros resultados (Tabela 1), dos 296 ensaios analisados, em 26,0\% houve significância do efeito de blocos, e em $43,6 \%$, do efeito de cultivares. Como a semeadura de cada um dos ensaios foi realizada com o mesmo número de sementes em todas as parcelas, não há justificativa para atribuir à causas aleatórias a significância do efeito de cultivares em tantos ensaios. Logo, existe uma diferenciação genética entre as cultivares quanto à mortalidade de plantas durante o desenvolvimento da cultura, e esse efeito é bem maior em cultivares de ciclo superprecoce do que em cultivares de ciclo normal (Tabela 1). A justificativa para tal fato é de que cultivares de ciclo superprecoce são semeadas quando a temperatura média do solo é menor, fazendo com que a germinação e o desenvolvimento inicial das plantas levem mais tempo, permitindo que os fatores genéticos de resistência das cultivares às intempéries se manifestem com maior freqüência.

Dos 296 ensaios analisados, em 54,4\% o efeito da população final de plantas sobre o rendimento de grãos não foi significativo, resultando no grupo Ys (Tabela 1), do qual foi estimada a porcentagem de ensaios com efeito de bloco, cultivar e as estatísticas DMS e HD. Nos outros $45,6 \%$ dos ensaios, o 
TABELA 1. Caracterização de 296 ensaios de competição de cultivares de milho conduzidos no Rio Grande do Sul nos anos de 1993 a 1996, por ciclo, por categoria e no geral, avaliando o efeito de blocos e de cultivares na população final de plantas $(X)$ e rendimento de grãos $(Y)$, a covariância de $Y$ com $X$, a diferença mínima significativa pelo teste de Tukey $(5 \%)$ em porcentagem da média (DMS) e a amplitude entre as médias estimadas dividida pelo valor do teste de Tukey (HD). Santa Maria, 1998.

\begin{tabular}{|c|c|c|c|c|c|c|c|}
\hline \multirow[t]{2}{*}{ Estatística } & \multicolumn{3}{|c|}{ Ciclo } & \multicolumn{3}{|c|}{ Categoria } & \multirow[t]{2}{*}{ Geral } \\
\hline & Superprecoce & Precoce & Normal & Preliminar & Estadual & Recomendado & \\
\hline № de ensaios (\%) & $99(33)$ & $106(36)$ & $91(31)$ & $54(18)$ & $166(56)$ & $76(26)$ & $296(100)$ \\
\hline X: $\%^{1}$ com efeito bloco & 27 & 29 & 21 & 22 & 24 & 33 & 26,0 \\
\hline X: $\%^{1}$ com efeito cultivar & 52 & 42 & 35 & 50 & 42 & 42 & 43,6 \\
\hline X: DMS & 18,6 & 20,8 & 21,8 & 23,7 & 19,2 & 19,3 & 20,2 \\
\hline Ys: Sem covariância $\left(\%^{1}\right)$ & 51,5 & 59,4 & 51,6 & 40,7 & 63,8 & 43,4 & 54,4 \\
\hline$\%$ com efeito bloco (Ys) & 51 & 55 & 28 & 68 & 41 & 48 & 46 \\
\hline$\%$ com efeito cultivar (Ys) & 86 & 95 & 83 & 86 & 91 & 82 & 89 \\
\hline DMS (Ys) & 30,4 & 30,7 & 23,9 & 35,4 & 27,5 & 28,8 & 28,8 \\
\hline HD (Ys) & 4,7 & 4,5 & 5,4 & 4,1 & 5,0 & 4,7 & 4,8 \\
\hline Yc: Com covariância $\left(\%^{1}\right)$ & 48,5 & 40,6 & 48,4 & 59,3 & 36,2 & 56,6 & 45,6 \\
\hline$\%$ com efeito bloco $(\mathrm{Yc})$ & 46 & 51 & 48 & 50 & 47 & 49 & 48,1 \\
\hline$\%$ com efeito cultivar (Yc) & 87 & 88 & 75 & 81 & 83 & 86 & 83,7 \\
\hline$\%$ com efeito cult. ajust.(Yc) & 85 & 88 & 89 & 87 & 85 & 91 & 87,4 \\
\hline DMS (Yc) & 31,5 & 34,1 & 28,7 & 39,5 & 28,4 & 30,3 & 31,6 \\
\hline DMS ajust. (Yc) & 29,8 & 32,2 & 28,8 & 38,3 & 26,6 & 29,2 & 30,2 \\
\hline $\mathrm{HD}(\mathrm{Yc})$ & 4,5 & 4,1 & 4,8 & 3,9 & 4,7 & 4,5 & 4,5 \\
\hline HD ajust. (Yc) & 18,3 & 16,7 & 19,2 & 14,3 & 19,9 & 18,4 & 18,1 \\
\hline Efi ciênci a relativa $(\%)$ & 115,2 & 119,5 & 130,6 & 123,7 & 120,9 & 121,2 & 121,7 \\
\hline
\end{tabular}

${ }^{1}$ Porcentual dos ensaios de cada ciclo e do geral.

efeito da população final de plantas sobre o rendimento de grãos (covariância) foi significativo a $5 \%$ de probabilidade, resultando no grupo Yc (Tabela 1), que, para fins de comparação, também foi analisado sem a covariância. Assim, observa-se que a porcentagem de ensaios com efeito de cultivar foi pouco afetada $(3,7 \%)$ com a aplicação do ajuste no efeito de cultivares quando a covariância é significativa. De forma semelhante, a estatística DMS reduziu pouco $(1,4 \%)$ com o uso da covariância quando ela foi significativa. Considerando que a média geral do ensaio não é afetada pelo ajuste das médias na análise da covariância, uma redução na DMS é devida a uma redução na estimativa ponderada da variância entre duas médias ajustadas de cultivares (Steel \& Torrie, 1960), já que o grau de liberdade do erro perde apenas uma unidade. Por outro lado, no grupo Yc, a estatística HD passou de 4,5 (sem aplicar a análise da covariância) para 18,1 com a aplicação da análise da covariância quando ela foi significativa. A causa da alteração na estatística HD, certamente, é o aumento na amplitude entre as médias como conseqüência de seu ajustamento. Observações semelhantes podem ser obtidas nos diferentes ciclos de cultivares e nas diferentes categorias de ensaios (Tabela 1).

Quanto à variável rendimento de grãos, o efeito de blocos foi significativo em $46 \%$ dos ensaios analisados sem análise da covariância, e em $48,1 \%$ com análise da covariância, o que justifica o uso do delineamento em blocos ao acaso na totalidade dos ensaios de competição de cultivares.

O efeito de cultivar foi significativo em $89 \%$ sem análise da covariância, e em $87,4 \%$ com análise da covariância dos ensaios, sem maiores diferenças entre os ciclos e as categorias (Tabela 1) dos ensaios. A não-significância em $11 \%$ dos ensaios sem covariância e em 12,6\% com covariância poderia ser justificada pelo alto erro experimental e pela criteriosa seleção das cultivares, que é feita anualmente para compor os ensaios.

A estatística que melhor indica a vantagem do uso da análise da covariância é a HD, a qual apresenta um desempenho médio quatro vezes superior em relação ao não-uso da covariância. Essa melhoria da qualidade dos resultados acarreta melhor discriminação entre as médias quando são ajustadas para 
uma mesma população final de plantas. A dificuldade reside em saber se a cultivar A é superior à $\mathrm{B}$ em virtude de melhor desempenho durante o desenvolvimento e maturação ou menor perda de plantas na germinação e estádio inicial do desenvolvimento, que também pode ser resultante de fatores genéticos das cultivares. Lopes (1993) concluiu que o uso da análise da covariância reduz a DMS entre cultivares de milho, porém esses resultados foram obtidos principalmente com o uso da covariável número de espigas, que se mostrou significativa com maior freqüência do que a covariável população final de plantas. Como as variáveis população final de plantas e número de espigas, geralmente, são correlacionadas e possivelmente dependentes das cultivares, o uso dessas covariáveis para controle do erro experimental pode ficar prejudicado e com isso, sugerem-se novos estudos para definir a covariável a ser utilizada.

Os indicativos de qualidade dos ensaios, tais como a DMS, HD e eficiência relativa são mais favoráveis quando existe paralelismo entre as cultivares na resposta da produção de grãos em função da popula-

TABELA 2. Caracterização de 296 ensaios de competição de cultivares de milho conduzidos no Rio Grande do Sul nos anos de 1993 a 1996, classificados quanto ao efeito da covariância e paralelismo entre cultivares da relação de $Y$ (rendimento de grãos) com $X$ (população final de plantas), avaliando o efeito de blocos e de cultivares, a diferença mínima significativa pelo teste de Tukey $(5 \%)$ em porcentagem da média (DMS), e a amplitude entre as médias estimadas dividida pelo valor do teste de Tukey (HD). Santa Maria, 1998.

\begin{tabular}{lccc}
\hline Estatística & $\begin{array}{c}\text { Sem } \\
\text { covariância }\end{array}$ & \multicolumn{2}{c}{ Com covariância } \\
\cline { 3 - 4 } & & Paralelo & Não paralelo \\
\hline Ne de ensaios (\%) & $161(54,3)$ & $85(28,7)$ & $50(16,9)$ \\
\% com efe ito cultivar & 88,2 & 82,3 & 86,0 \\
\% com efeito cult. ajustado & - & 87,0 & 88,0 \\
DMS & 28,8 & 29,3 & 35,2 \\
DMS ajustado & - & 28,7 & 32,8 \\
HD & 4,8 & 4,7 & 4,0 \\
HD ajustado & - & 19,5 & 15,6 \\
Eficiência relativa (\%) & - & 124,3 & 117,1 \\
\hline & & \multicolumn{2}{c}{}
\end{tabular}

ção final de plantas (Tabela 2), conforme exigência do método de análise de covariância. Dos ensaios em que a covariância foi significativa, em 37\% não há paralelismo, isto é, a resposta da produção de grãos à população final de plantas não é a mesma nas diferentes cultivares avaliadas. Algumas cultivares são mais influenciadas do que outras. Nesses casos, o ajuste das médias não é adequado.

\section{CONCLUSÕES}

1. A estatística DMS (diferença mínima significativa) obtida pelo teste de Tukey expresso em porcentagem da média é inferior à estatística HD (amplitude entre as médias dividido pela valor do teste de Tukey) para expressar o poder de discriminação entre cultivares.

2. A análise da covariância aumenta o poder de discriminação entre cultivares de milho.

\section{AGRADECIMENTOS}

Aos engenheiros agrônomos Miguel Bresolin, Ronaldo Matzenauer e a outros técnicos da FEPAGRO/RS, pela disponibilização dos dados analisados.

\section{REFERÊNCIAS}

BOX, G.E.P.; COX, D.R. An analysis of transformations. Royal Statistical Society Journal, Series B, Oxford, n.26, p.211-252, 1964.

CAMPOS, H. Estatística experimental nãoparamétrica. 4.ed. Piracicaba: ESALQ-Departamento de Matemática e Estatística, 1983. 349p.

COCHRAN, W.G. Some consequences when the assumptions for the analysis of variance are not satisfied. Biometrics, Washington, v.3, p.22-38, 1947.

ESTEFANEL, V.; PIGNATARO, I.A.B.; STORCK, L. Avaliação do coeficiente de variação de experimentos com algumas culturas agrícolas. In: SIMPÓSIO DE ESTATÍSTICA APLICADA À EXPERIMENTAÇÃO AGRONÔMICA, 2., 1987, Londrina. Anais. Londrina : Universidade Estadual de Londrina, 1987. p.115-131.

Pesq. agropec. bras., Brasília, v.35, n.7, p.1311-1316, jul. 2000 
LOPES, S.J. Avaliação do efeito de diferentes formas de adubação sobre a precisão de ensaios de milho. Santa Maria : UFSM, 1993. 72p. Dissertação de Mestrado.

LOPES, S.J.; STORCK, L. A precisão experimental para diferentes manejos na cultura do milho. Ciência Rural, Santa Maria, v.25, n.1, p.49-53, 1995.

LÚCIO, A.D. Parâmetros da precisão experimental das principais culturas anuais do Estado do Rio Grande do Sul. Santa Maria : UFSM, 1997. 64p. Dissertação de Mestrado.

OLIVEIRA, P.H. de. Tamanho e forma ótimos da parcela para avaliação do rendimento em experimentos com batata. Santa Maria : UFSM, 1994. 83p. Dissertação de Mestrado.

PIMENTEL-GOMES, F. Curso de estatística experimental. 13.ed. Piracicaba : Nobel, 1990. p.383-401.
SEBER, G.A.F. Linear regression analysis. New York : J. Wiley, 1976. 465p.

SIQUEIRA, A.L. Uso de transformação em análise de variância e análise de regressão. São Paulo : USP, 1983. 154p. Dissertação de Mestrado.

STEEL, R.G.D.; TORRIE, J.H. Principles and procedures of statistics. New York : McGraw Hill, 1960. 481p.

STORCK, L. Estimativa para tamanho e forma de parcelas e número de repetições para experimentos com milho (Zea mays L.). Porto Alegre : UFRGS, 1979. 98p. Dissertação de Mestrado.

STORCK, L.; LOPES, S.J. Experimentação II. Santa Maria : UFSM-Departamento de Fitotecnia, 1997. $217 \mathrm{p}$ 